\title{
Clades and clans: a comparison study of two evolutionary models
}

\author{
Sha Zhu · Cuong Than · Taoyang Wu
}

Received: date / Accepted: date

\begin{abstract}
The Yule-Harding-Kingman (YHK) model and the proportional to distinguishable arrangements (PDA) model are two binary tree generating models that are widely used in evolutionary biology. Understanding the distributions of clade sizes under these two models provides valuable insights into macro-evolutionary processes, and is important in hypothesis testing and Bayesian analyses in phylogenetics. Here we show that these distributions are log-convex, which implies that very large clades or very small clades are more likely to occur under these two models. Moreover, we prove that there exists a critical value $\kappa(n)$ for each $n \geqslant 4$ such that for a given clade with size $k$, the probability that this clade is contained in a random tree with $n$ leaves generated under the YHK model is higher than that under the PDA model if $1<k<\kappa(n)$, and lower if $\kappa(n)<k<n$. Finally, we extend our results to binary unrooted trees, and obtain similar results for the distributions of clan sizes.
\end{abstract}

Keywords Phylogenetic trees $\cdot$ Null models $\cdot$ Clade $\cdot$ Clan $\cdot$ Log-convexity

\section{Introduction}

Distributions of genealogical features such as shapes, subtrees, and clades are of interest in phylogenetic and population genetics. By comparing biological data with these distributions, which can be derived from null models such as the YuleHarding-Kingman (YHK) model and proportional to distinguishable arrangements (PDA) model, we can obtain insights into macro-evolutionary processes underlying the data (Felsenstein, 2004; Mooers and Heard, 1997, 2002; Nordborg,

SZ was supported in part by the New Zealand Marsden Fund, CT by the National Science Foundation contract DBI-1146722, and TW by the Singapore MOE grant R-146-000-134-112.

Sha Zhu

Wellcome Trust Centre for Human Genetics, University of Oxford, United Kingdom

E-mail: sha.joe.zhu@gmail.com

Cuong Than

Department of Computer Science, University of Tuebingen, Germany

E-mail: thvcuong@gmail.com

Taoyang Wu $(\varangle)$

School of Computing Sciences, University of East Anglia, United Kingdom

E-mail: taoyang.wu@gmail.com 
1998, 2001). For instance, phylogenetic tree statistics were used to study variation in speciation and extinction rates (see, e.g. Agapow and Purvis (2002); Mooers and Heard (1997); Rogers (1996)).

As a basic concept in phylogenetic studies and systematic classification of species, a clade, also known as a monophyletic group, is a subset of extant species containing all the descendants of a common ancestor. In this paper, we are interested in the distributions of clade size in a random tree generated under the null models. Such distributions have been utilized in hypothesis testing as to whether a set of extant taxa forms a clade (Hudson and Coyne, 2002; Rosenberg, 2007), and are relevant to the Bayesian approach to phylogenetic reconstruction (Pickett and Randle, 2005; Steel and Pickett, 2006).

Two well-studied and commonly used null models in evolutionary biology are the Yule-Harding model (Yule, 1925; Harding, 1971) and the PDA model (also known as the uniform model) (Aldous, 2001). Loosely speaking, under the PDA model all rooted binary trees are chosen with equal probabilities, while under the Yule-Harding model each tree is chosen with a probability proportion to the number of total orders that can be assigned to internal nodes of the tree so that the relative (partial) order is preserved (see, e.g. Semple and Steel, 2003). More precisely, the Yule-Harding model assumes a speciation process with a constant pure-birth rate (Blum et al, 2006; Pinelis, 2003), which generates the same probability distributions of tree topologies as Kingman's coalescent process (Kingman, 1982). Therefore, we will refer to it as the Yule-Harding-Kingman (YHK) model (Aldous, 1996). Both the YHK model and PDA model are used to generate prior probabilities of tree topologies in Bayesian phylogenetic analyses (Li et al, 2000; Rannala and Yang, 1996).

Comparison studies of various tree statistics between the YHK and PDA models have been reported in the literature. For example, McKenzie and Steel (2000) derive the asymptotic probability distributions of cherries in phylogenetic trees; Steel (2012) discusses the root location in a random Yule or PDA tree; Blum et al (2006) obtain formulas for the mean, variance, and covariance of the Sackin (Sackin, 1972) and Colless (Colless, 1982) indices, two popular indices used to measure the balance of phylogenetic trees.

Note that in Bayesian analyses, the output is often clade support calculated from the consensus of the approximated posterior distribution of the topologies. However, the relationships between topological priors and clade priors are often not straightforward. For instance, it is observed that the uniform topological prior, which is induced by the PDA model, leads to non-uniform clade priors (Pickett and Randle, 2005). Indeed, for $n>4$, neither the PDA model nor the YHK model gives rise to a uniform prior on clades (Steel and Pickett, 2006). As an attempt to further elucidate these relationships, in this paper we study the distributions of clade sizes in the PDA model, and then conduct a comparison study of these distributions with those in the YHK model. In addition, we conduct a similar study on clans, the counterpart of clades for unrooted trees.

The remainder of the paper is organized as follows. Sections 2 and 3 contain necessary notation and background used in the paper and a brief review of the YHK and PDA models. We then present in Section 4 the results concerning clade probabilities under the two null models, and those related to clan probabilities in Section 5. Finally, we conclude in Section 6 with discussions and remarks. 

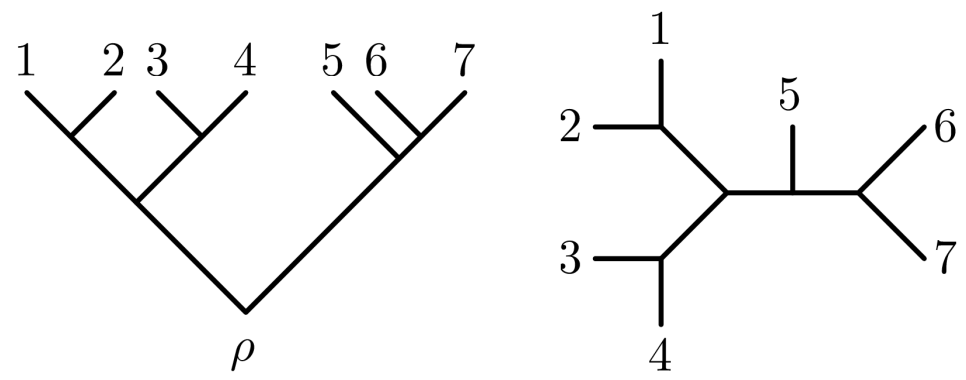

Fig. 1 Example of a rooted phylogenetic tree (left) and an unrooted phylogenetic tree (right).

\section{Preliminaries}

In this section, we present some basic notation and background concerning phylogenetic trees and log-convexity that will be used in this paper. From now on, $X$ will be used to denote the leaf set, and we assume that $X$ is a finite set of size $n=|X| \geqslant 3$ unless stated otherwise.

\subsection{Phylogenetic trees}

A tree is a connected acyclic graph. A vertex will be referred to as a leaf if its degree is one, and an interior vertex otherwise. An unrooted tree is binary if all interior vertices have degree three. A rooted tree is a tree that has exactly one distinguished node designated as the root, which is usually denoted by $\rho$. A rooted tree is binary if the root has degree two and all other interior vertices have degree three.

A phylogenetic tree on $X$ is a binary tree with leaves bijectively labeled by elements of $X$. The set of rooted and unrooted phylogenetic trees on $X$ are denoted by $\mathscr{T}_{X}$ and $\mathscr{T}_{X}^{*}$, respectively. Two examples of phylogenetic trees on $X=\{1, \ldots, 7\}$, one rooted and the other unrooted, are presented in Figure 1.

Let $T$ be a rooted phylogenetic tree on $X$. Given two vertices $v$ and $u$ in tree $T, u$ is below $v$ if $v$ is contained in the path between $u$ and the root of $T$. In this case, we also say $u$ is a descendant of $v$ if $v$ and $u$ are distinct. A clade of $T$ is a subset of $X$ that contains precisely all the leaves below a vertex in $T$. A clade $A$ is called trivial if $|A|=1$ or $|A|=X$ holds, and non-trivial otherwise. Since $T$ has $2 n-1$ vertices, it contains precisely $2 n-1$ clades, including $n+1$ trivial ones. For example, the rooted phylogenetic tree on $X=\{1, \ldots, 7\}$ depicted in Figure 1 has 13 clades: the five non-trivial ones are $\{1,2\},\{3,4\},\{1,2,3,4\},\{6,7\}$ and $\{5,6,7\}$.

Suppressing the root of a tree $T$ in $\mathscr{T}_{X}$, that is, removing $\rho$ and replacing the two edges incident with $\rho$ with an edge connecting the two vertices adjacent to $\rho$, results in an unrooted tree in $\mathscr{T}_{X}^{*}$, which will be denote by $\rho^{-1}(T)$. For instance, for the rooted tree $T$ and unrooted tree $T^{*}$ in Figure 1 , we have $T^{*}=\rho^{-1}(T)$. Note that for each $T^{*}$ in $\mathscr{T}_{X}^{*}$, there are precisely $2 n-3$ rooted trees $T$ in $\mathscr{T}_{X}$ such that $T^{*}=\rho^{-1}(T)$ holds.

Recall that a split $A \mid B$ on $X$ is a bipartition of $X$ into two disjoint non-empty sets $A$ and $B$, that is, $A \cap B=\emptyset$ and $A \cup B=X$. Let $T^{*}$ be an unrooted tree in $\mathscr{T}_{X}^{*}$. Every edge $e$ of $T^{*}$ induces a necessarily unique split $A \mid B$ of $X$ obtained as the two sets of leaves separated by $e$. In other words, the path between a pair of leaves in $X$ contains $e$ if and only if one of these two leaves is in $A$ and the other one is in $B$. In this case, we say $A \mid B$ is a split contained in $T^{*}$. A $c l a n A$ of 
$T^{*}$ is a subset of $X$ such that $A \mid(X \backslash A)$ is a split contained in $T^{*}$. Since $T^{*}$ has $2 n-3$ edges and each edge induces two distinct clans, it contains precisely $2(2 n-3)$ clans.

\subsection{Log-convexity}

A sequence $\left\{y_{1}, \ldots, y_{m}\right\}$ of real numbers is called positive if each number contained in the sequence is greater than zero. It is called log-convex if $y_{k-1} y_{k+1} \geqslant y_{k}^{2}$ holds for $2 \leqslant k \leqslant m-1$. Clearly, a positive sequence $\left\{y_{k}\right\}_{1 \leqslant k \leqslant m}$ is log-convex if and only if the sequence $\left\{y_{k+1} / y_{k}\right\}_{1 \leqslant k \leqslant m-1}$ is increasing. Therefore, a log-convex sequence of positive numbers is necessarily unimodal, that is, there exists an index $1 \leqslant k \leqslant m$ such that

$$
y_{1} \geqslant y_{2} \geqslant \cdots \geqslant y_{k} \text { and } y_{k} \leqslant y_{k+1} \leqslant \cdots \leqslant y_{m}
$$

hold. Recall that a sequence $\left\{y_{i}\right\}_{1 \leqslant i \leqslant m}$ is also called unimodal if $y_{1} \leqslant y_{2} \leqslant \cdots \leqslant y_{k}$ and $y_{k} \geqslant y_{k+1} \geqslant \cdots \geqslant y_{m}$ hold for some $1 \leqslant k \leqslant m$. However, in this paper, unimodal is always referred to the situation specified in Eq. (1).

For later use, we end this section with the following results concerning log-convex sequences (see, e.g. Liu and Wang (2007)).

Lemma 1 If $\left\{y_{i}\right\}_{1 \leqslant i \leqslant m}$ and $\left\{y_{i}^{\prime}\right\}_{1 \leqslant i \leqslant m}$ are two positive and log-convex sequences, then the sequences $\left\{y_{i}+y_{i}^{\prime}\right\}_{1 \leqslant i \leqslant m}$ and $\left\{y_{i} \cdot y_{i}^{\prime}\right\}_{1 \leqslant i \leqslant m}$ are positive and log-convex.

\section{The PDA and YHK models}

In this section, we present a formal definition of the two null models investigated in this paper: the proportional to distinguishable arrangements (PDA) model and Yule-Harding-Kingman (YHK) model.

To begin with, recall that the number of rooted phylogenetic trees with leaf set $X$ with $n=|X|$ is

$$
\varphi(n):=(2 n-3) ! !=1 \cdot 3 \cdots(2 n-3)=\frac{(2 n-2) !}{2^{n-1}(n-1) !} .
$$

Here we will use the convention that $\varphi(1)=1$. Under the PDA model, each tree has the same probability to be generated, that is, we have

$$
\mathbb{P}_{\mathrm{PDA}}(T)=\frac{1}{\varphi(n)}
$$

for every $T$ in $\mathscr{T}_{X}$.

Under the Yule-Harding model, a rooted phylogenetic tree on $X$ is generated as follows. Beginning with a two leafed tree, we "grow" it by repeatedly splitting a leaf into two new leaves. The splitting leaf is chosen randomly and uniformly among all the present leaves in the current tree. After obtaining an unlabeled tree with $n$ leaves, we label each of its leaves with a label sampled randomly uniformly (without replacement) from $X$. When branch lengths are ignored, the Yule-Harding model is shown by Aldous (1996) to be equivalent to the trees generated by Kingman's coalescent process, and so we call it the YHK model. Under this model, the probability of generating a tree $T$ in $\mathscr{T}_{X}$ is (Semple and Steel, 2003):

$$
\mathbb{P}_{\text {YHK }}(T)=\frac{2^{n-1}}{n !} \prod_{v \in V(T)} \frac{1}{\lambda_{v}}
$$


where $\stackrel{\circ}{V}(T)$ is the set of interior nodes of $T$, and $\lambda_{v}$ is the number of interior nodes of $T$ that are below $v$. For example, the probability of the rooted tree in Figure 1 is $2^{7-1} /(7 ! \times 3 \times 2 \times 6)$.

For an unrooted tree $T^{*}$ in $\mathscr{T}_{X}^{*}$, let $\rho\left(T^{*}\right)$ denote the set of rooted trees $T$ in $\mathscr{T}_{X}$ with $T^{*}=\rho^{-1}(T)$. As noted previously in Section 2, $T^{*}$ can be obtained from each of the $2 n-3$ rooted trees $T$ in $\rho\left(T^{*}\right)$ by removing the root of $T$. Using this correspondence scheme, a probability measure $\mathbb{P}$ on $\mathscr{T}_{X}$ induces a probability measure $\mathbb{P}_{u}$ on the set $\mathscr{T}_{X}^{*}$. That is, we have

$$
\mathbb{P}_{u}\left(T^{*}\right)=\sum_{T \in \rho\left(T^{*}\right)} \mathbb{P}(T)
$$

In particular, let $\mathbb{P}_{\mathrm{uYHK}}$ and $\mathbb{P}_{\mathrm{uPDA}}$ denote the probability measures on $\mathscr{T}_{X}^{*}$ induced by $\mathbb{P}_{\mathrm{YHK}}$ and $\mathbb{P}_{\mathrm{PDA}}$, respectively. Note that this implies

$$
\mathbb{P}_{\mathrm{uPDA}}\left(T^{*}\right)=\frac{1}{\varphi(n-1)}
$$

for every $T^{*}$ in $\mathscr{T}_{X}^{*}$. Since the number of unrooted phylogenetic trees on $X$ is $\left|\mathscr{T}_{X}^{*}\right|=\varphi(n-1)=(2 n-5) !$ !, each tree in $\mathscr{T}_{X}^{*}$ has the same probability under $\mathbb{P}_{\mathrm{uPDA}}$.

We end this section with a property of the PDA and YHK models that will play an important role in obtaining our results. Recall that a probability measure $\mathbb{P}$ on $\mathscr{T}_{X}$ has the exchangeability property if $\mathbb{P}$ depends only on tree shapes, that is, if two rooted trees $T^{\prime}$ and $T$ can be obtained from each other by permuting their leaves, then $\mathbb{P}(T)=\mathbb{P}\left(T^{\prime}\right)$ holds. Similarly, a probability measure on $\mathscr{T}_{X}^{*}$ has the exchangeability property if it depends only on tree shapes. It is wellknown that both $\mathbb{P}_{\text {YHK }}$ and $\mathbb{P}_{\text {PDA }}$, the probability measures on the set of rooted trees $\mathscr{T}_{X}$ induced by the YHK and PDA models, have the exchangeability property (Aldous, 1996), By Eqs. (5) and (4), we can conclude that the probability measures $\mathbb{P}_{\mathrm{uYHK}}$ and $\mathbb{P}_{\mathrm{uPDA}}$ on the set of unrooted trees $\mathscr{T}_{X}^{*}$ also have the exchangeability property.

\section{Clade probabilities}

In this section, we shall present our main results on clade probabilities. To this end, we need some further notation and definitions. Given a rooted binary tree $T$, let

$$
\mathbb{I}_{T}(A)= \begin{cases}1, & \text { if } A \text { is a clade of } T \\ 0, & \text { otherwise }\end{cases}
$$

be the 'indicator' function that maps a subset $A$ of $X$ to 1 if $A$ is a clade of $T$, and 0 otherwise. Now for a subset $A$ of $X$, the probability of $X$ being a clade of a random tree sampled according to a probability distribution $\mathbb{P}$ on $\mathscr{T}_{X}$ is defined as

$$
\mathbb{P}(A)=\sum_{T \in \mathscr{T}_{X}} \mathbb{P}(T) \mathbb{I}_{T}(A)
$$

Since $\sum_{A \subseteq X} \mathbb{I}_{T}(A)=2 n-1$ for each $T \in \mathscr{T}_{X}$ and $\sum_{T \in \mathscr{T}_{X}} \mathbb{P}(T)=1$, we have

$$
\sum_{A \subseteq X} \mathbb{P}(A)=\sum_{A \subseteq X} \sum_{T \in \mathscr{T}_{X}} \mathbb{P}(T) \mathbb{I}_{T}(A)=\sum_{T \in \mathscr{T}_{X}} \mathbb{P}(T) \sum_{A \subseteq X} \mathbb{I}_{T}(A)=2 n-1
$$

By the last equation, we note that each probability measure $\mathbb{P}$ on $\mathscr{T}_{X}$ induces a measure on the set of all subsets of $X$, which can be normalized to a probability measure by a factor of $1 /(2 n-1)$. 
The above definitions on a subset of $X$ can be extended to a collection of subsets of $X$. That is, given a collection of subsets $\left\{A_{1}, \ldots, A_{k}\right\}$ of $X$, we have

$$
\mathbb{I}_{T}\left(A_{1}, \ldots, A_{m}\right)=\mathbb{I}_{T}\left(A_{1}\right) \cdots \mathbb{I}_{T}\left(A_{m}\right)
$$

and

$$
\mathbb{P}\left(A_{1}, \ldots, A_{m}\right)=\sum_{T \in \mathscr{T}_{X}} \mathbb{P}(T)\left(\mathbb{I}_{T}\left(A_{1}\right) \cdots \mathbb{I}_{T}\left(A_{m}\right)\right)
$$

Note that $\mathbb{I}_{T}\left(A_{1}, \ldots, A_{m}\right)=1$ if and only if each $A_{i}$ is a clade of $T$ for $1 \leqslant i \leqslant m$. On the other hand, it is well known (see, e.g. Semple and Steel (2003)) that given a collection of subsets $\left\{A_{1}, \ldots, A_{k}\right\}$ of $X$, there exists a tree $T \in \mathscr{T}_{X}$ with $\mathbb{I}_{T}\left(A_{1}, \ldots, A_{m}\right)=1$ if and only if $\left\{A_{1}, \ldots, A_{k}\right\}$ forms a hierarchy, that is, $A_{i} \cap A_{j} \in\left\{\emptyset, A_{i}, A_{j}\right\}$ holds for $1 \leqslant i<j \leqslant m$.

The following result shows that if a probability measure depends only on tree shapes, then the clade probabilities derived from it are also independent of the 'labeling' of the elements.

Lemma 2 Let $\mathbb{P}$ be a probability measure on $\mathscr{T}_{X}$ that has the exchangeability property. Then for each pair of subsets $A$ and $A^{\prime}$ of $X$ with $|A|=\left|A^{\prime}\right|$, we have

$$
\mathbb{P}(A)=\mathbb{P}\left(A^{\prime}\right) \text { and } \quad \mathbb{P}(A, X \backslash A)=\mathbb{P}\left(A^{\prime}, X \backslash A^{\prime}\right)
$$

Proof Suppose that $A$ and $A^{\prime}$ are two subsets of $X$ that have the same size. Then there exists a permutation $\pi$ on $X$ such that $A^{\prime}=A^{\pi}:=\{\pi(x) \mid x \in A\}$. Now for each tree $T$ in $\mathscr{T}_{X}$, let $T^{\pi}$ be the tree obtained from $T$ by relabeling the leaves of $T$ according to permutation $\pi$. Then $A$ is a clade of $T$ if and only if $A^{\pi}$ is a clade of $T^{\pi}$. Together with Eq. (7), we have

$$
\begin{aligned}
\mathbb{P}(A) & =\sum_{T \in \mathscr{T}_{X}} \mathbb{P}(T) \mathbb{I}_{T}(A)=\sum_{T \in \mathscr{T}_{X}} \mathbb{P}(T) \mathbb{I}_{T^{\pi}}\left(A^{\pi}\right) \\
& =\sum_{T \in \mathscr{T}_{X}} \mathbb{P}\left(T^{\pi}\right) \mathbb{I}_{T^{\pi}}\left(A^{\pi}\right)=\sum_{T^{\pi} \in \mathscr{T}_{X}} \mathbb{P}\left(T^{\pi}\right) \mathbb{I}_{T^{\pi}}\left(A^{\pi}\right)=\mathbb{P}\left(A^{\pi}\right),
\end{aligned}
$$

where the third equality follows from the exchangeability property of $\mathbb{P}$. This shows $\mathbb{P}(A)=\mathbb{P}\left(A^{\prime}\right)$, and a similar argument leads to $\mathbb{P}(A, X \backslash A)=\mathbb{P}\left(A^{\prime}, X \backslash A^{\prime}\right)$.

Since $\mathbb{P}_{\text {YHK }}$ has the exchangeability property, by Lemma 2 we know that $\mathbb{P}_{\text {YHK }}(A)$ is determined by the size of $A$ only. Therefore, we denote

$$
p_{n}(a)=\mathbb{P}_{\mathrm{YHK}}(A)
$$

as the probability that a random tree in $\mathscr{T}_{X}$, where $n=|X|$, induces a specific clade $A$ of size $a$ under the YHK model. Similarly, we let

$$
q_{n}(a)=\mathbb{P}_{\mathrm{PDA}}(A)
$$

be the probability that a random tree in $\mathscr{T}_{X}$ induces a specific clade $A$ of size $a$ under the PDA model. In addition, we also denote

$$
p_{n}(a, n-a)=\mathbb{P}_{\mathrm{YHK}}(A, X \backslash A), \quad \text { and } \quad q_{n}(a, n-a)=\mathbb{P}_{\mathrm{PDA}}(A, X \backslash A),
$$

the probabilities that both $A$ and $X \backslash A$ are clades of a tree in $\mathscr{T}_{X}$ generated under the YHK and PDA models, respectively. Note that if both $A$ and $X \backslash A$ are clades of a tree $T$, then they are precisely the clades consisting of the leaves below the two children of the root of $T$. 
Corollary 1 Let $\mathbb{P}$ be a probability measure on $\mathscr{T}_{X}$ that has the exchangeability property. For each $1 \leqslant a \leqslant n$, the expected number of clades with size a contained in a random tree sampled according to $\mathbb{P}$ is

$$
\left(\begin{array}{l}
n \\
a
\end{array}\right) \mathbb{P}(A)
$$

where $A$ is an arbitrary subset of $X$ with $|A|=a$.

Proof Denote the collection of subsets of $X$ with size $a$ by $\mathscr{X}_{a}$ and fix a subset $A \in \mathscr{X}_{a}$. Let $Z_{T}(a):=\sum_{Y \in \mathscr{X}_{a}} \mathbb{I}_{T}(Y)$ be the number of clades with size $a$ contained in a tree $T$. Then the expected number of clades with size $a$ contained in a random tree sampled according to $\mathbb{P}$ is given by

$$
\sum_{T \in \mathscr{T}_{X}} \mathbb{P}(T) Z_{T}(a)=\sum_{T \in \mathscr{T}_{X}} \sum_{Y \in \mathscr{X}_{a}} \mathbb{P}(T) \mathbb{I}_{T}(Y)=\sum_{Y \in \mathscr{X}_{a}} \sum_{T \in \mathscr{T}_{X}} \mathbb{P}(T) \mathbb{I}_{T}(Y)=\sum_{Y \in \mathscr{X}_{a}} \mathbb{P}(Y)=\left(\begin{array}{l}
n \\
a
\end{array}\right) \mathbb{P}(A),
$$

where the last equality holds because by Lemma 2 we have $\mathbb{P}(Y)=\mathbb{P}(A)$ for all $Y \in \mathscr{X}_{a}$.

\subsection{Clade probabilities under the YHK model}

In this subsection we study the clade probabilities under the YHK model. First, we have the following theorem concerning the computation of $p_{n}(a)$ and $p_{n}(a, n-a)$, which was discovered and rediscovered several times in the literature (see, e.g., Blum and Francois (2005); Brown (1994); Heard (1992); Rosenberg (2003, 2006)).

Theorem 1 For a positive integer $a \leqslant n-1$ we have:

(i) $p_{n}(a)=\frac{2 n}{a(a+1)}\left(\begin{array}{l}n \\ a\end{array}\right)^{-1}$.

(ii) $p_{n}(a, n-a)=\frac{2}{n-1}\left(\begin{array}{l}n \\ a\end{array}\right)^{-1}$.

By the above results, we show below that clade probabilities under the YHK model form a log-convex sequence. This implies that the clades with small or large size are more likely to be generated than those with middle size under the model.

Theorem 2 For $n \geqslant 3$, the sequence $\left\{p_{n}(a)\right\}_{1 \leqslant a \leqslant n}$ and $\left\{p_{n}(a, n-a)\right\}_{1 \leqslant a<n}$ are log-convex. Moreover, let

$$
\Delta(n):=\sqrt{n+\left(\frac{n-3}{4}\right)^{2}}+\frac{n-3}{4}
$$

then we have

(i) $p_{n}(a) \geqslant p_{n}(a+1)$ for $a \leqslant \Delta(n)$, and $p_{n}(a)<p_{n}(a+1)$ for $a>\Delta(n)$, and

(ii) $p_{n}(a, n-a)>p_{n}(a+1, n-a-1)$ for $a \leqslant n / 2$ and $p_{n}(a, n-a)<p_{n}(a+1, n-a-1)$ for $a \geqslant n / 2$.

Proof Let $y_{a}=\frac{2 n}{a(a+1)}$ for $1 \leqslant a \leqslant n-1$ and $y_{n}=1$, and $y_{a}^{\prime}=\left(\begin{array}{l}n \\ a\end{array}\right)^{-1}$ for $1 \leqslant a \leqslant n$. Since $\left\{y_{a}\right\}_{1 \leqslant a \leqslant n}$ and $\left\{y_{a}^{\prime}\right\}_{1 \leqslant a \leqslant n}$ are both log-convex, by Lemma 1 and Theorem 1 we can conclude that the sequence $\left\{p_{n}(a)\right\}_{1 \leqslant a \leqslant n}$ is log-convex. A similar argument shows that $\left\{p_{n}(a, n-a)\right\}_{1 \leqslant a<n}$ is also log-convex.

By Theorem 1, we have

$$
\frac{p_{n}(a+1)}{p_{n}(a)}=\frac{a(a+1)\left(\begin{array}{l}
n \\
a
\end{array}\right)}{(a+1)(a+2)\left(\begin{array}{c}
n \\
a+1
\end{array}\right)}=\frac{a(a+1)}{(a+2)(n-a)},
$$

for $1 \leqslant a \leqslant n-2$. The last equation is less than or equal to 1 if and only if

$$
a(a+1) \leqslant(a+2)(n-a) \Longleftrightarrow 2 a^{2}-(n-3) a-2 n \leqslant 0 .
$$

Therefore, $p_{n}(a+1) \leqslant p_{n}(a)$ if and only if $a \leqslant \Delta(n)$. This establishes Part (i) of the theorem.

Part (ii) of the theorem follows from the fact that $\left(\begin{array}{l}n \\ a\end{array}\right)<\left(\begin{array}{c}n \\ a+1\end{array}\right)$ for $a \leqslant n / 2$ and $\left(\begin{array}{l}n \\ a\end{array}\right)>\left(\begin{array}{c}n \\ a+1\end{array}\right)$ for $a \geqslant n / 2$. 
4.2 Clade probabilities under the PDA model

Parallel to those in the Section 4.1, in this subsection we derive results on clade probabilities under the PDA model.

Theorem 3 For a positive integer $a \leqslant n-1$ we have:

(i) $q_{n}(a)=\frac{\varphi(a) \varphi(n-a+1)}{\varphi(n)}=\left(\begin{array}{c}n-1 \\ a-1\end{array}\right)\left(\begin{array}{c}2 n-2 \\ 2 a-2\end{array}\right)^{-1}$.

(ii) $q_{n}(a, n-a)=\frac{\varphi(a) \varphi(n-a)}{\varphi(n)}=\frac{1}{(2 n-2 a-1)}\left(\begin{array}{c}n-1 \\ a-1\end{array}\right)\left(\begin{array}{c}2 n-2 \\ 2 a-2\end{array}\right)^{-1}$.

Proof To derive the formula for $q_{n}(a)$, it suffices to show that there are $\varphi(a) \varphi(n-a+1)$ trees in $\mathscr{A}$, the subset of trees in $\mathscr{T}_{X}$ containing $A$ as a clade, because the probability of each tree in $\mathscr{T}_{X}$ is $1 / \varphi(n)$. Without loss of generality, we can assume that $X=\{1,2, \cdots, n\}$ and $A=\{n-a+1, \cdots, n\}$. Let

$$
X^{\prime}:=(X-A) \cup\{n-a+1\}=\{1,2, \cdots, n-a, n-a+1\}
$$

then each tree in $\mathscr{A}$ can be generated by the following two steps: picking up a tree in $\mathscr{T}_{X^{\prime}}$ and replacing the leaf with label $n-a+1$ by a tree from $\mathscr{T}_{A}$. In addition, a different choice of trees in the first step or the second step will result in a different tree in $\mathscr{A}$. Since there are $\varphi(n-a+1)$ possible choices in the first step and $\varphi(a)$ ones in second step, we can conclude that the number of trees $\mathscr{A}$ is $\varphi(a) \varphi(n-a+1)$. In addition, using the fact that

$$
\varphi(m)=(2 m-3) ! !=\frac{(2 m-2) ! !}{2^{m-1}(m-1) !}
$$

holds for $m \geqslant 1$, we have

$$
q_{n}(a)=\frac{\varphi(a) \varphi(n-a+1)}{\varphi(n)}=\frac{(2 a-2) !(2 n-2 a) !(n-1) !}{(2 n-2) !(a-1) !(n-a) !}=\left(\begin{array}{c}
n-1 \\
a-1
\end{array}\right)\left(\begin{array}{c}
2 n-2 \\
2 a-2
\end{array}\right)^{-1} .
$$

The proof of the formula for $q_{n}(a, n-a)$ is similar to the one for $q_{n}(a)$. Let $\mathscr{A}^{*}$ be the collection of the trees in $\mathscr{T}_{X}$ containing both $A$ and $X-A$ as clades. Then a tree in $\mathscr{A}^{*}$ is uniquely determined by choosing a tree in $\mathscr{T}_{A}$, and subsequently another tree from $\mathscr{T}_{X-A}$. This implies the number of trees in $\mathscr{A}^{*}$ is $\varphi(a) \varphi(n-a)$. Hence

$$
\begin{aligned}
q_{n}(a, n-a) & =\frac{\varphi(a) \varphi(n-a)}{\varphi(n)}=\frac{1}{(2 n-2 a-1)} q_{n}(a) \\
& =\frac{1}{(2 n-2 a-1)}\left(\begin{array}{c}
n-1 \\
a-1
\end{array}\right)\left(\begin{array}{l}
2 n-2 \\
2 a-2
\end{array}\right)^{-1} .
\end{aligned}
$$

Recall that in Theorem 2 we show that clade probabilities under the YHK model form a log-convex sequence. Here we establish a similar result for the PDA model, which implies that the sequences $\left\{q_{n}(a)\right\}_{1 \leqslant a<n}$ and $\left\{q_{n}(a, n-a)\right\}_{1 \leqslant a<n}$ are also unimodal.

Theorem 4 For $n \geqslant 3$, the sequence $\left\{q_{n}(a)\right\}_{1 \leqslant a \leqslant n}$ and $\left\{q_{n}(a, n-a)\right\}_{1 \leqslant a<n}$ are log-convex. Moreover, we have

(i) $q_{n}(a+1) \geqslant q_{n}(a)$ when $a \geqslant n / 2$, and $q_{n}(a+1) \leqslant q_{n}(a)$ when $a \leqslant n / 2$.

(ii) $q_{n}(a+1, n-a-1) \geqslant q_{n}(a, n-a)$ when $a \geqslant(n-1) / 2$, and $q_{n}(a+1, n-a-1) \geqslant q_{n}(a, n-a)$ when $a \leqslant(n-1) / 2$. 
Proof By Theorem 3 and $q_{n}(n)=1$, for $1 \leqslant a<n$ we have

$$
\frac{q_{n}(a+1)}{q_{n}(a)}=\frac{2 a-1}{2 n-2 a-1},
$$

which is greater than or equal to 1 when $2 a-1 \geqslant 2 n-2 a-1$, or equivalently when $a \geqslant n / 2$. Thus Part (i) follows. Moreover, we have

$$
\frac{q_{n}(a+1) q_{n}(a-1)}{q_{n}^{2}(a)}=\left(\frac{2 a-1}{2 a-3}\right)\left(\frac{2 n-2 a+1}{2 n-2 a-1}\right) \geqslant 1,
$$

for $2 \leqslant a<n$, and hence $\left\{q_{n}(a)\right\}_{1 \leqslant a \leqslant n}$ is log-convex.

Similarly, we have

$$
\frac{q_{n}(a+1, n-a-1)}{q_{n}(a, n-a)}=\left(\frac{2 n-2 a-1}{2 n-2 a-3}\right)\left(\frac{q_{n}(a+1)}{q_{n}(a)}\right)=\frac{2 a-1}{2 n-2 a-3},
$$

which is greater than or equal to 1 when $2 a-1 \geqslant 2 n-2 a-3$, or equivalently when $a \geqslant(n-1) / 2$. Moreover, we have

$$
\frac{q_{n}(a+1, n-a-1) q_{n}(a-1, n-a+1)}{q_{n}^{2}(a, n-a)}=\left(\frac{2 a-1}{2 a-3}\right)\left(\frac{2 n-2 a-1}{2 n-2 a-3}\right) \geqslant 1,
$$

and hence $\left\{q_{n}(a)\right\}_{1 \leqslant a<n}$ is log-convex.

\subsection{A comparison between the PDA and YHK models}

Using the formulae for computing clade probabilities under the PDA and YHK models presented in the previous two subsections, here we investigate the differences between these two models. Let's begin with comparing $p_{n}(a)$ and $q_{n}(a)$, the probabilities of a specific (and fixed) clade of size $a$ under the YHK and PDA models, respectively. As an example, consider the ratio of $p_{n}(a) / q_{n}(a)$ with $n=30$ as depicted in Figure 2. Then it is clear that, except for $a=1$ for which both $p_{n}(a)=q_{n}(a)=1$, the ratio is strictly decreasing and is less than 1 when $a$ is greater than certain value. This "phase transition' type phenomenon holds for all $n>3$, as the following theorem shows.

Theorem 5 For $n>3$, there exists a number $\kappa(n)$ in $[2, n-1]$, such that $p_{n}(a)>q_{n}(a)$ for $2 \leqslant a<\kappa(n)$, and $p_{n}(a)<$ $q_{n}(a)$ for $\kappa(n)<a \leqslant n-1$.

Proof Let

$$
g_{n}(a)=\frac{p_{n}(a)}{q_{n}(a)}=\frac{2 n}{a(a+1)}\left(\begin{array}{c}
2 n-2 \\
2 a-2
\end{array}\right)\left(\begin{array}{l}
n \\
a
\end{array}\right)^{-1}\left(\begin{array}{c}
n-1 \\
a-1
\end{array}\right)^{-1}
$$

Using the identity $\left(\begin{array}{c}m \\ k+1\end{array}\right)=\frac{m-k}{k+1}\left(\begin{array}{c}m \\ k\end{array}\right)$, we obtain

$$
\frac{g_{n}(a+1)}{g_{n}(a)}=\frac{a(a+1)(2 n-2 a-1)}{(a+2)(2 a-1)(n-a)} .
$$

We have

$$
a(a+1)(2 n-2 a-1)<(a+2)(2 a-1)(n-a) \Longleftrightarrow a>\frac{2 n}{n+3},
$$

and hence $g_{n}(a)>g_{n}(a+1)$ for $2 n /(n+3)<a \leqslant n-2$. Since $2 n /(n+3)<2$, we have $g_{n}(2)>g_{n}(3)>\cdots>g_{n}(n-1)$.

It is easy to see that for $n>3$,

$$
g_{n}(2)=\frac{2(2 n-3)}{3(n-1)}>1
$$

and

$$
g_{n}(n-1)=\frac{2(2 n-3)}{n(n-1)}<1
$$

This and the fact that $g_{n}(a)$ is strictly decreasing on $[2, n-1]$ imply the existence of the number $\kappa(n)$ in the theorem. 

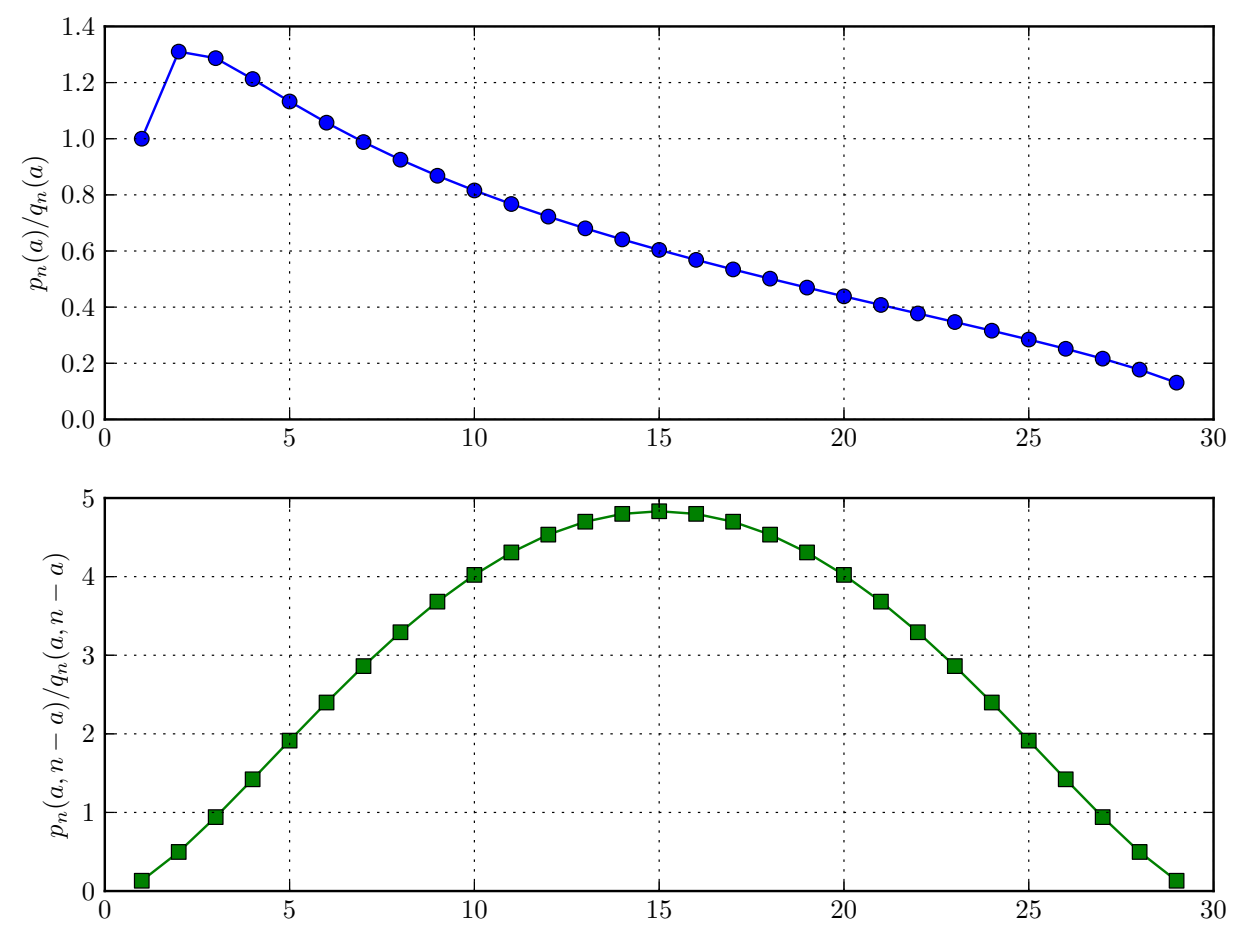

Fig. 2 Plots of the ratios $p_{n}(a) / q_{n}(a)$ and $p_{n}(a, n-a) / q_{n}(a, n-a)$, with $n=30$ and $a=1, \ldots, 29$.

Next, we consider $p_{n}(a, n-a)$ and $q_{n}(a, n-a)$. Note that by definition, both $p_{n}(a)$ and $q_{n}(a, n-a)$ are symmetric about $n / 2$, as demonstrated by the plot of the ratio $p_{n}(a, n-a) / q_{n}(a, n-a)$ with $n=30$ in Figure 2 . In addition, the figure shows that the ratio is strictly increasing on the interval $[1,\lfloor n / 2\rfloor]$ (and by the symmetry of the ratio, it is strictly decreasing on the interval $[\lceil n / 2\rceil, n-1])$. This observation is made precise and rigorous in the following theorem.

Theorem 6 For $n>3$, there exists a number $\lambda(n)$ in $[1,\lfloor n / 2\rfloor]$, such that $p_{n}(a, n-a)<q_{n}(a, n-a)$ for $1 \leqslant a \leqslant \lambda(n)$, and $p_{n}(a, n-a)>q_{n}(a, n-a)$ for $\lambda(n)<a \leqslant\lfloor n / 2\rfloor$.

Proof Let

$$
h_{n}(a)=\frac{p_{n}(a, n-a)}{q_{n}(a, n-a)}=\frac{2(2 n-2 a-1)}{n-1}\left(\begin{array}{l}
2 n-2 \\
2 a-2
\end{array}\right)\left(\begin{array}{l}
n \\
a
\end{array}\right)^{-1}\left(\begin{array}{l}
n-1 \\
a-1
\end{array}\right)^{-1} .
$$

Then

$$
\frac{h_{n}(a+1)}{h_{n}(a)}=\frac{(a+1)(2 n-2 a-3)}{(2 a-1)(n-a)}>1,
$$

where the last inequality follows from the observation that

$$
(a+1)(2 n-2 a-3)-(n-a)(2 a-1)=3(n-2 a-1)>0
$$

holds for $1 \leqslant a \leqslant\lfloor n / 2\rfloor-1$. This implies that the function $h_{n}(a)$ is strictly increasing on the interval $[1,\lfloor n / 2\rfloor]$.

Thus, it now suffices to show that $h_{n}(1) \leqslant 1$ and $h_{n}(\lfloor n / 2\rfloor) \geqslant 1$ in order to demonstrate the existence of $\lambda(n)$. We have

$$
h_{n}(1)=\frac{p_{n}(1, n-1)}{q_{n}(1, n-1)}=\frac{2(2 n-3)}{n(n-1)}<1,
$$

if $n>3$. Let $k=\lfloor n / 2\rfloor$. If $n$ is even (i.e., $k=n / 2$ ), then for $k \geqslant 2$

$$
\begin{aligned}
h_{2 k}(k) & =\frac{2(4 k-2 k-1)}{(2 k-1)}\left(\begin{array}{c}
4 k-2 \\
2 k-2
\end{array}\right)\left(\begin{array}{c}
2 k \\
k
\end{array}\right)^{-1}\left(\begin{array}{c}
2 k-1 \\
k-1
\end{array}\right)^{-1} \\
& =\left(\begin{array}{c}
4 k-2 \\
2 k-2
\end{array}\right)\left(\begin{array}{c}
2 k-1 \\
k-1
\end{array}\right)^{-2}>1 .
\end{aligned}
$$




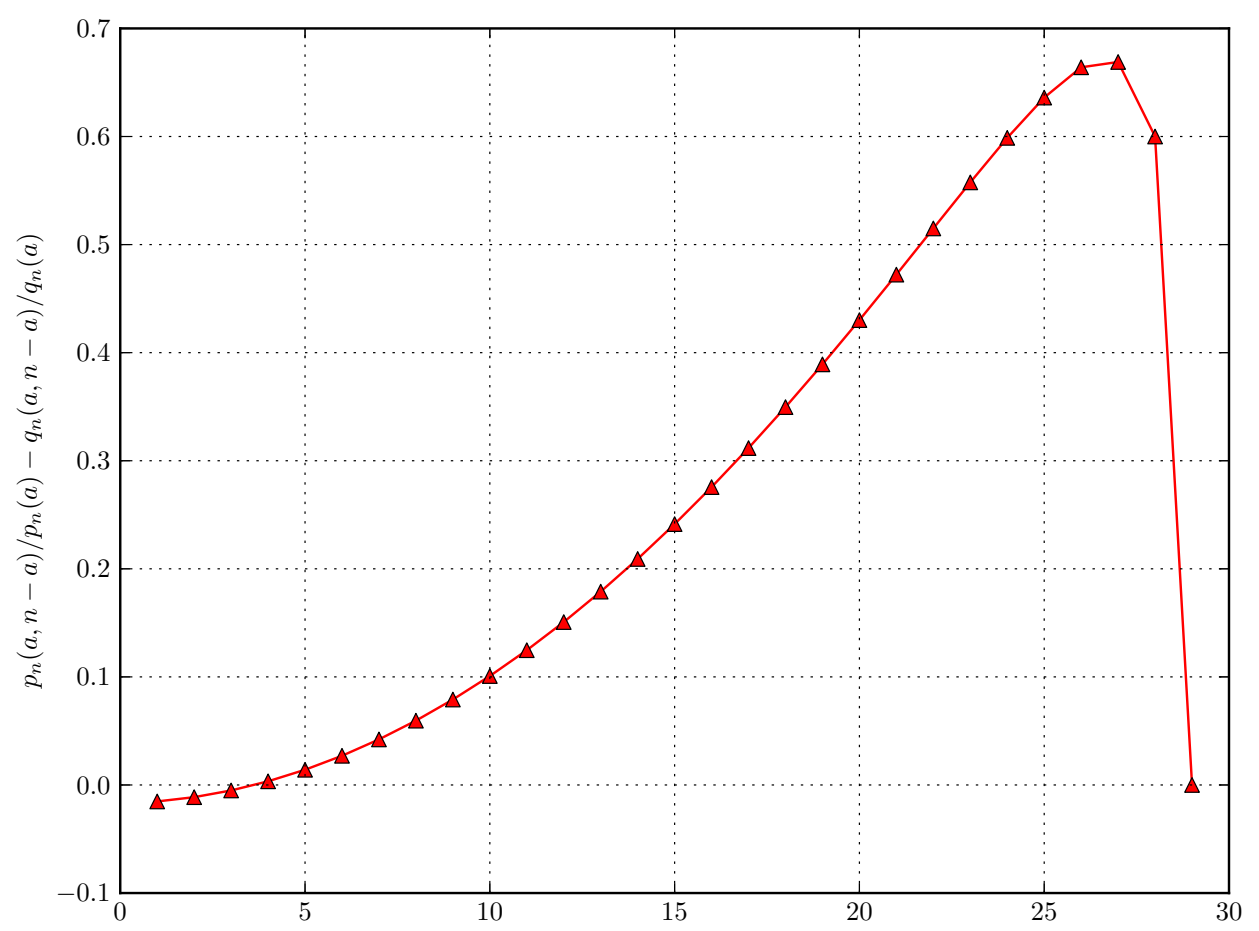

Fig. 3 Plot of function $u_{n}(a)$ with $n=30$.

The inequality in the last equation can be seen as follows. Let $A$ and $B$ be two sets, each having $(2 k-1)$ elements. The number of subsets of $A \cup B$ that have $k-1$ elements from each of $A$ and $B$ is $\left(\begin{array}{c}2 k-1 \\ k-1\end{array}\right)^{2}$. On the other hand, the total number of $(2 k-2)$-subsets of $A \cup B$ is $\left(\begin{array}{c}4 k-2 \\ 2 k-2\end{array}\right)$.

If $n$ is odd (i.e., $k=(n-1) / 2)$, then

$$
\begin{aligned}
h_{2 k+1}(k) & =\frac{2(2 k+1)}{2 k}\left(\begin{array}{c}
4 k \\
2 k-2
\end{array}\right)\left(\begin{array}{c}
2 k+1 \\
k
\end{array}\right)^{-1}\left(\begin{array}{c}
2 k \\
k-1
\end{array}\right)^{-1} \\
& =\frac{2 k+1}{k}\left(\begin{array}{c}
4 k \\
2 k-2
\end{array}\right) \frac{k}{2 k+1}\left(\begin{array}{c}
2 k \\
k-1
\end{array}\right)^{-2} \\
& =\left(\begin{array}{c}
4 k \\
2 k-2
\end{array}\right)\left(\begin{array}{c}
2 k \\
k-1
\end{array}\right)^{-2} .
\end{aligned}
$$

Using the same argument as in proving $h_{2 k}(k)>1$, we also have $h_{2 k+1}(k) \geqslant 1$ for $k \geqslant 1$.

Let $A$ be a fixed subset of $X$ with size $a$, where $1 \leqslant a \leqslant n-1$. In the previous two theorems, we present comparison results for $\mathbb{P}(A)$ and $\mathbb{P}(A, X \backslash A)$ under the YHK and PDA models. We end this subsection with a comparison study of $\mathbb{P}(A, X \backslash A) / \mathbb{P}(A)$, that is, the probability that a tree $T \in \mathscr{T}_{X}$ sampled according to probability measure $\mathbb{P}$ contains both $A$ and $X \backslash A$ as its clades (which means that $A$ and $X \backslash A$ are the clades below the two children of the root of $T$ ), given that $A$ is a clade of $T$. To this end, let

$$
u_{n}(a)=\frac{p_{n}(a, n-a)}{p_{n}(a)}-\frac{q_{n}(a, n-a)}{q_{n}(a)}=\frac{a(a+1)}{n(n-1)}-\frac{1}{2 n-2 a-1}
$$

be the difference between the two conditional probabilities under the two models. We are interested in the sign changes of $u_{n}(a)$ as it indicates a 'phase transitions' between these two models. For instance, considering the values of $u_{n}(a)$ for $n=30$ as depicted in Figure 3, then there exists a unique change of sign. Indeed, the observation that there exists a unique change of sign of $u_{n}(a)$ holds for general $n$, as the following theorem shows. 
Theorem 7 For $n \geqslant 3$, there exists $\tau(n) \in[1, n-1]$ such that $u_{n}(a) \leqslant 0$ if $a \leqslant \tau(n)$ and $u_{n}(a) \geqslant 0$ if $a \geqslant \tau(n)$.

Proof Consider the function

$$
f_{n}(x)=\frac{x(x+1)}{n(n-1)}-\frac{1}{2 n-2 x-1}, \quad x \in \mathbb{R} .
$$

Clearly $f_{n}(x)$ agrees with $u_{n}(a)$ when $x=a$. Then

$$
f_{n}^{\prime}(x)=\frac{2 x+1}{n(n-1)}-\frac{2}{(2 n-2 x-1)^{2}}=\frac{t(2 n-t)^{2}-2 n(n-1)}{n(n-1)(2 n-t)^{2}},
$$

where $t=2 x+1$. The sign of $f_{n}^{\prime}(x)$ thus depends on the sign of

$$
g_{n}(t)=t(2 n-t)^{2}-2 n(n-1)
$$

We see that $g_{n}(t)$ is a polynomial of $t$ of degree 3, and hence it can have at most three (real) roots. On the other hand, for $n \geqslant 3$, we have:

$$
\begin{gathered}
g_{n}(0)=-2 n(n-1)<0, \\
g_{n}(1)=n^{2}+(n-1)^{2}>0, \\
g_{n}(2 n-1)=-2 n(n-2)-1<0,
\end{gathered}
$$

and

$$
\lim _{t \rightarrow \infty} g_{n}(t)=\infty
$$

Therefore, $g_{n}(t)$ has exactly three roots $t_{1} \in(0,1), t_{2} \in(1,2 n-1)$, and $t_{3}>2 n-1$. Note further that $g_{n}(n)=n^{3}-$ $2 n(n-1)=n\left((n-1)^{2}+1\right)>0$, and hence $t_{2}>n$. Denoting $x_{i}=\left(t_{i}-1\right) / 2$ for $1 \leqslant i \leqslant 3$, then we have $f_{n}^{\prime}(x)=0$ for $x \in\left\{x_{1}, x_{2}, x_{3}\right\}, f_{n}^{\prime}(x)<0$ for $x \in\left(-\infty, x_{1}\right) \cup\left(x_{2}, x_{3}\right)$, and $f_{n}^{\prime}(x)>0$ for $x \in\left(x_{1}, x_{2}\right) \cup\left(x_{3}, \infty\right)$. Since $x_{1}=\left(t_{1}-1\right) / 2<0$ and $f_{n}(a)=u_{n}(a)$, the sign of $f_{n}^{\prime}(x)$ implies that $u_{n}(1)<u_{n}(2)<\cdots<u_{n}\left(\left\lfloor x_{2}\right\rfloor\right)$. Similarly, we also have $u_{n}\left(\left\lceil x_{2}\right\rceil\right)>$ $\cdots>u_{n}(n-2)>u_{n}(n-1)$. It is easy to see that for $n \geqslant 3$

$$
\begin{gathered}
u_{n}(1)=\frac{2}{n(n-1)}-\frac{1}{2 n-3}=-\frac{(n-2)(n-3)}{n(n-1)(2 n-3)} \leqslant 0 \\
u_{n}(n-1)=\frac{n(n-1)}{n(n-1)}-\frac{1}{2 n-2(n-1)-1}=0 .
\end{gathered}
$$

Since $x_{2}=\left(t_{2}-1\right) / 2<n-1$ and $x_{3}=\left(t_{3}-1\right) / 2>n-1,\left\lceil x_{2}\right\rceil \leqslant n-1<x_{3}$. This implies that $u_{n}\left(\left\lceil x_{2}\right\rceil\right)>\cdots>$ $u_{n}(n-2)>u_{n}(n-1)=0$. Therefore, there exists a positive number $\tau(n) \in\left[1, x_{2}\right]$ such that $u_{n}(a) \leqslant 0$ if $a \leqslant \tau(n)$ and $u_{n}(a) \geqslant 0$ if $a \geqslant \tau(n)$.

\subsection{Correlation results on the PDA model}

In this section, we generalize results in Section 4.2 for a collection of disjoint subsets of $X$, and then show that the two indicator variables $\mathbb{I}_{T}(A)$ and $\mathbb{I}_{T}(B)$ are positively correlated.

Theorem 8 Let $A_{1}, \ldots, A_{k}$ be $k$ disjoint (nonempty) subsets of $X$. Denoting $\left|A_{1}\right|+\cdots+\left|A_{k}\right|$ by $m$, then we have

$$
\mathbb{P}_{P D A}\left(A_{1}, \ldots, A_{k}\right)=\frac{\varphi(n-m+k) \prod_{i=1}^{k} \varphi\left(\left|A_{i}\right|\right)}{\varphi(n)} .
$$


Proof We first compute the number of trees that have $A_{1}, \ldots, A_{k}$ as clades. To this end, note that such a tree can be constructed in two steps:

1. Build a tree on $\left(X \backslash \bigcup_{i=1}^{k} A_{i}\right) \cup\left\{x_{1}, \ldots, x_{k}\right\}$, where $x_{1}^{\prime}, \ldots, x_{k}^{\prime}$ are leaves not in $X$ serving as "placeholders" used in the second step.

2. Replace each $x_{i}^{\prime}$ with a tree in $\mathscr{T}_{A_{i}}$.

There are $\varphi(n-m+k)$ different choices for a tree in the first step, and $\prod_{i=1}^{k} \varphi\left(\left|A_{i}\right|\right)$ different ways to replace $x_{1}^{\prime}, \ldots, x_{k}^{\prime}$ by trees in $\mathscr{T}_{A_{1}}, \ldots, \mathscr{T}_{A_{k}}$ in the second step. Therefore the number of trees that have $A_{1}, \ldots, A_{k}$ as clades is $\varphi(n-m+$ k) $\prod_{i=1}^{k} \varphi\left(\left|A_{i}\right|\right)$. Together with the fact that each tree in $\mathscr{T}_{X}$ is chosen with probability $1 / \varphi(n)$ under the PDA model, this implies the theorem.

Note that $\left|A_{1}\right|+\cdots+\left|A_{k}\right|=n$ when $A_{1}, \ldots, A_{k}$ form a partition of $X$. Therefore, we obtain the following result as a simple consequence of Theorem 8 (see Theorem 5.1 in Zhu et al (2011) for a parallel result on the YHK model).

Corollary 2 If $A_{1}, \ldots, A_{k}$ form a partition of $X$, then

$$
\mathbb{P}_{P D A}\left(A_{1}, \ldots, A_{k}\right)=\frac{\varphi(k) \prod_{i=1}^{k} \varphi\left(\left|A_{i}\right|\right)}{\varphi(n)} .
$$

Theorem 8 is a general result concerning a collection of clades. When there are only two clades, the below theorem provides a more detailed analysis.

Theorem 9 Let $A$ and $B$ be two subsets of $X$ with $a \leqslant b$, where $a=|A|$ and $b=|B|$. Then we have

$$
\mathbb{P}_{P D A}(A, B)= \begin{cases}\frac{\varphi(a) \varphi(n-b+1) \varphi(b-a+1)}{\varphi(n)}, & \text { if } A \subseteq B, \\ \frac{\varphi(a) \varphi(b) \varphi(n-a-b+2)}{\varphi(n)}, & \text { if } A \text { and } B \text { are disjoint } \\ 0, & \text { otherwise. }\end{cases}
$$

Proof The first case follows by applying Theorem 2 twice. The second case is a special case of Theorem 8 . The third case holds because if $A \cap B \notin\{A, B, \emptyset\}$, then there exists no tree that contains both $A$ and $B$ as its clades.

To establish the last result of this subsection, we need the following technical lemma.

Lemma 3 Let $m, n, m^{\prime}, n^{\prime}$ be positive numbers with $\left(m-m^{\prime}\right)\left(n-n^{\prime}\right) \geqslant 0$, then

$$
\varphi\left(m^{\prime}+n^{\prime}\right) \varphi(m+n) \geqslant \varphi\left(m+n^{\prime}\right) \varphi\left(m^{\prime}+n\right) .
$$

In particular, if $a \leqslant b \leqslant b^{\prime} \leqslant a^{\prime}$ are positive numbers with $a+a^{\prime}=b+b^{\prime}$, then we have

$$
\varphi(a) \varphi\left(a^{\prime}\right) \geqslant \varphi(b) \varphi\left(b^{\prime}\right)
$$

Proof To establish the first claim, we may assume $m \geqslant m^{\prime}$ and $n \geqslant n^{\prime}$, as the proof of the other case, $m \leqslant m^{\prime}$ and $n \leqslant n^{\prime}$, is similar. Now Eqn. (11) holds because we have

$$
\begin{aligned}
\frac{\varphi(m+n)}{\varphi\left(m+n^{\prime}\right)} & =\frac{(2(m+n)-3) \cdot(2(m+n)-5) \cdots 3 \cdot 1}{\left(2\left(m+n^{\prime}\right)-3\right) \cdot\left(2\left(m+n^{\prime}\right)-5\right) \cdots 3 \cdot 1} \\
& =(2 m+2 n-3)(2 m+2 n-5) \cdots\left(2 m+2 n^{\prime}+1\right)\left(2 m+2 n^{\prime}-1\right) \\
& \geqslant\left(2 m^{\prime}+2 n-3\right)\left(2 m^{\prime}+2 n-5\right) \cdots\left(2 m^{\prime}+2 n^{\prime}+1\right)\left(2 m^{\prime}+2 n^{\prime}-1\right) \\
& =\frac{\varphi\left(m^{\prime}+n\right)}{\varphi\left(m^{\prime}+n^{\prime}\right)}
\end{aligned}
$$


Here Eq. (13) follows from $n \geqslant n^{\prime}$ and Eq. (14) from $m \geqslant m^{\prime}$.

The second assertion follows from the first one by setting $m^{\prime}=n^{\prime}=a / 2, m=b-a / 2$ and $n=b^{\prime}-a / 2$.

We end this section with the following result, which says that the random variables $\mathbb{I}_{T}(A)$ and $\mathbb{I}_{T}(B)$ are positively correlated when $A$ and $B$ are compatible, that is, $A \cap B \in\{\emptyset, A, B\}$.

Theorem 10 Let $A$ and $B$ be two compatible non-empty subsets of $X$; then

$$
\mathbb{P}_{P D A}(A, B) \geqslant \mathbb{P}_{P D A}(A) \mathbb{P}_{P D A}(B) .
$$

Proof Set $a=|A|$ and $b=|B|$. By symmetry we may assume without loss of generality that $a \leqslant b$ holds. Since $A$ and $B$ are compatible, we have either $A \cap B=\emptyset$ or $A \subseteq B$.

Since $n-a-b+2 \leqslant n-b+1 \leqslant n-a+1 \leqslant n$, by Lemma 3 we have

$$
\varphi(n) \varphi(n-a-b+2) \geqslant \varphi(n-b+1) \varphi(n-a+1),
$$

and hence

$$
\frac{\varphi(a) \varphi(b) \varphi(n-a-b+2)}{\varphi(n)} \geqslant \frac{\varphi(b) \varphi(n-b+1)}{\varphi(n)} \frac{\varphi(a) \varphi(n-a+1)}{\varphi(n)} .
$$

Together with Theorem 9, this shows that the theorem holds for the case $A \cap B=\emptyset$.

On the other hand, noting that $b-a+1 \leqslant b \leqslant n$ and $b-a+1 \leqslant n-a+1 \leqslant n$ holds, by Lemma 3 we have

$$
\varphi(n) \varphi(b-a+1) \geqslant \varphi(b) \varphi(n-a+1),
$$

and hence

$$
\frac{\varphi(a) \varphi(b-a+1)}{\varphi(b)} \frac{\varphi(b) \varphi(n-b+1)}{\varphi(n)} \geqslant \frac{\varphi(b) \varphi(n-b+1)}{\varphi(n)} \frac{\varphi(a) \varphi(n-a+1)}{\varphi(n)} .
$$

Together with Theorem 9, this shows that the theorem holds for the case $A \subseteq B$, as required.

\section{Clan probabilities}

In this section, we study clan probabilities, the counterpart of clade probabilities for unrooted trees. To this end, given a subset $A \subseteq X$ and an unrooted tree $T^{*} \in \mathscr{T}_{X}^{*}$, let $\mathbb{I}_{T^{*}}(A)$ be the indicator function defined as

$$
\mathbb{I}_{T^{*}}(A)= \begin{cases}1, & \text { if } A \text { is a clan of } T^{*}, \\ 0, & \text { otherwise. }\end{cases}
$$

Then the probability that clan $A$ is contained in a random unrooted tree sampled according to $\mathbb{P}_{u}$ is

$$
\mathbb{P}_{u}(A)=\sum_{T^{*} \in \mathscr{T}_{X}^{*}} \mathbb{P}_{u}\left(T^{*}\right) \mathbb{I}_{T^{*}}(A)
$$

Note that the the clan probability defined as above can be extended to a collection of subsets in a natural way, that is, we have

$$
\mathbb{P}_{u}\left(A_{1}, \ldots, A_{m}\right)=\sum_{T^{*} \in \mathscr{T}_{X}^{*}} \mathbb{P}_{u}\left(T^{*}\right)\left(\mathbb{I}_{T^{*}}\left(A_{1}\right) \cdots \mathbb{I}_{T^{*}}\left(A_{m}\right)\right) .
$$

As a generalization of Lemma 6.1 in Zhu et al (2011), the following technical result relates clan probabilities to clade probabilities. 
Lemma 4 Suppose that $\mathbb{P}$ is a probability measure on $\mathscr{T}_{X}$ and $\mathbb{P}_{u}$ is the probability measure on $\mathscr{T}_{X}^{*}$ induced by $\mathbb{P}$. Then for a nonempty subset $A \subset X$, we have

$$
\mathbb{P}_{u}(A)=\mathbb{P}(A)+\mathbb{P}(X \backslash A)-\mathbb{P}(A, X \backslash A) .
$$

Proof It is well-known (see, e.g., Lemma 6.1 in Zhu et al (2011)) that for a rooted binary tree $T$, a set $A$ is a clan of $\rho^{-1}(T)$ if and only if either $A$ is a clade of $T$ or $X \backslash A$ is a clade of $T$. Now the lemma follows from the definitions and the inclusion-exclusion principle.

Now we proceed to studying the clan probabilities under the YHK and PDA models. To begin with, recall that the probabilities of an unrooted tree $T^{*} \in \mathscr{T}_{X}^{*}$ under the YHK and PDA models are

$$
\mathbb{P}_{\mathrm{uYHK}}\left(T^{*}\right)=\sum_{T \in \rho\left(T^{*}\right)} \mathbb{P}_{\mathrm{YHK}}(T) \text { and } \mathbb{P}_{\mathrm{uPDA}}\left(T^{*}\right)=\sum_{T \in \rho\left(T^{*}\right)} \mathbb{P}_{\mathrm{PDA}}(T),
$$

where $\rho\left(T^{*}\right)$ denotes the set of rooted trees $T$ in $\mathscr{T}_{X}$ with $T^{*}=\rho^{-1}(T)$.

By the definition of clan probabilities, we have

$$
\begin{aligned}
& \mathbb{P}_{\mathrm{uYHK}}(A)=\sum_{T^{*} \in \mathscr{T}_{X}^{*}} \mathbb{P}_{\mathrm{uYHK}}\left(T^{*}\right) \mathbb{I}_{T^{*}}(A), \text { and } \\
& \mathbb{P}_{\mathrm{uPDA}}(A)=\sum_{T^{*} \in \mathscr{T}_{X}^{*}} \mathbb{P}_{\mathrm{uPDA}}\left(T^{*}\right) \mathbb{I}_{T^{*}}(A) .
\end{aligned}
$$

It can be verified, as with the case of clade probabilities, that the exchangeability property of $\mathbb{P}_{\mathrm{uYHK}}$ and $\mathbb{P}_{\mathrm{uPDA}}$ implies that both $\mathbb{P}_{\mathrm{uYHK}}(A)$ and $\mathbb{P}_{\mathrm{uPDA}}(A)$ depend only on the size $a=|A|$, not on the particular elements in $A$. Therefore, we will denote them as $p_{n}^{*}(a)$ and $q_{n}^{*}(a)$, respectively.

By Lemma 4, we can derive the following formulae to calculate clan probabilities under the two models, the first of which is established in Zhu et al (2011). Note that the second formula reveals an interesting relationship between clan probability and clade probability under the PDA model. Intuitively, it is related to the observation that there exists a bijective mapping from $\mathscr{T}_{X}$ to $\mathscr{T}_{Y}^{*}$ with $Y=X \cup\{y\}$ for some $y \notin X$ that maps each rooted tree $T$ in $\mathscr{T}_{X}$ to the unique tree in $\mathscr{T}_{Y}^{*}$ obtained from $T$ by adding the leaf $y$ to the root of $T$.

Theorem 11 For $1 \leqslant a<n$, we have

$$
\begin{aligned}
p_{n}^{*}(a) & =2 n\left[\frac{1}{a(a+1)}+\frac{1}{(n-a)(n-a+1)}-\frac{1}{(n-1) n}\right]\left(\begin{array}{l}
n \\
a
\end{array}\right)^{-1} \\
q_{n}^{*}(a) & =\frac{\varphi(a) \varphi(n-a+1)+\varphi(n-a) \varphi(a+1)-\varphi(a) \varphi(n-a)}{\varphi(n)} \\
& =\frac{\varphi(a) \varphi(n-a)}{\varphi(n-1)}=q_{n-1}(a) .
\end{aligned}
$$

Proof Since the first equation is established in Zhu et al (2011), it remains to show the second one. The first equality follows from Lemma 4 and Theorem 3. To establish the second equality, it suffices to see that

$$
\begin{aligned}
\varphi(n-1)[ & \varphi(a) \varphi(n-a+1)+\varphi(n-a) \varphi(a+1)] \\
& =\varphi(n-1) \varphi(a) \varphi(n-a)[(2 n-2 a-1)+(2 a-1)] \\
& =\varphi(n-1)(2 n-2) \varphi(a) \varphi(n-a) \\
& =(\varphi(n)+\varphi(n-1)) \varphi(a) \varphi(n-a) .
\end{aligned}
$$




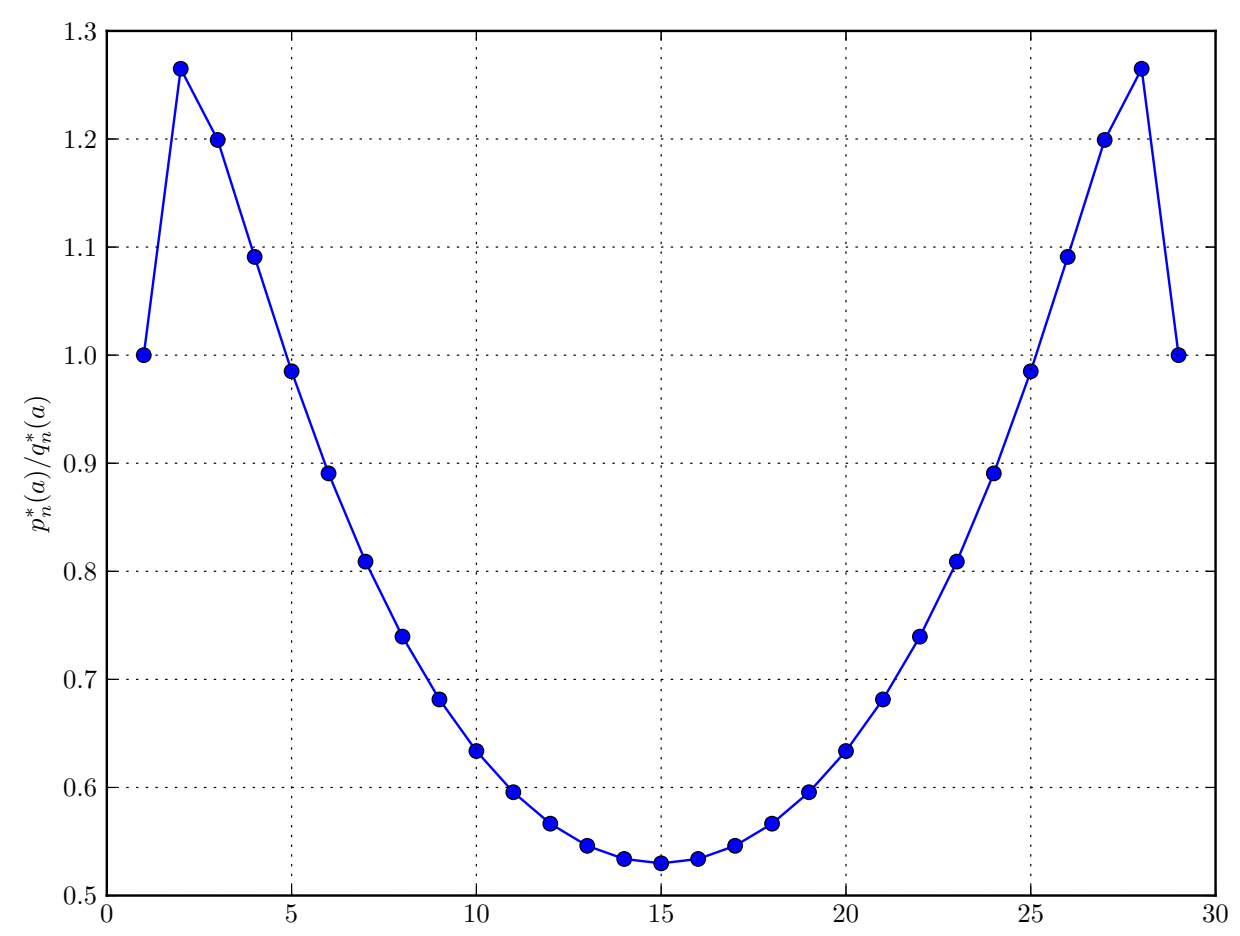

Fig. 4 Plot of the ratio $p_{n}^{*}(a) / q_{n}^{*}(a)$ with $n=30$ and $a=1, \ldots, 29$.

Recall that in Theorem 2 and 4 we show that the sequence $\left\{p_{n}(a)\right\}_{1 \leqslant a<n}$ and $\left\{q_{n}(a)\right\}_{1 \leqslant a<n}$ are log-convex. The theorem below establishes a similar result for clan probabilities.

Theorem 12 For $n \geqslant 3$, the sequence $\left\{p_{n}^{*}(a)\right\}_{1 \leqslant a<n}$ and $\left\{q_{n}^{*}(a)\right\}_{1 \leqslant a<n}$ are log-convex. Moreover, we have

(i) $p_{n}^{*}(a)=p_{n}^{*}(n-a)$ and $q_{n}^{*}(a)=q_{n}^{*}(n-a)$ for $1 \leqslant a<n$.

(ii) $q_{n}^{*}(a+1) \leqslant q_{n}^{*}(a)$ when $a \geqslant\lfloor(n-1) / 2\rfloor-1$, and $q_{n}^{*}(a+1) \geqslant q_{n}^{*}(a)$ when $a \leqslant\lceil(n-1) / 2\rceil$.

Proof Part (i) follows from Theorem 11. Since $q_{n}^{*}(a)=q_{n-1}(a)$ by Theorem 11, Part (ii) and that $\left\{q_{n}^{*}(a)\right\}_{1 \leqslant a<n}$ is log-convex follow from Theorem 4.

It remains to show that $\left\{p_{n}^{*}(a)\right\}_{1 \leqslant a<n}$ is log-convex. To this end, fix a number $n \geqslant 3$, and let $y_{a}=\frac{1}{a(a+1)}$ for $1 \leqslant$ $a<n$. Then clearly $\left\{y_{a}\right\}_{1 \leqslant a<n}$ is log-convex. This implies $\left\{y_{a}^{\prime}\right\}_{1 \leqslant a<n}$ with $y_{a}^{\prime}=y_{n-a}$ is also log-convex. In addition, since $2 y_{a} \geqslant y_{a+1}+y_{a-1}$ for $2 \leqslant a \leqslant n-2,\left\{y_{a}^{*}\right\}_{1 \leqslant a<n}$ with $y_{a}^{*}=y_{a}-\frac{1}{n(n-1)}$ is log-convex as well. By Lemma 1 , we know $\left\{y_{a}^{\prime}+y_{a}^{*}\right\}_{1 \leqslant a<n}$ is log-convex. As $\left\{\left(\begin{array}{l}n \\ a\end{array}\right)^{-1}\right\}_{1 \leqslant a<n}$ is log-convex, by Lemma 1 and Theorem 11 we conclude that $\left\{p_{n}^{*}(a)\right\}_{1 \leqslant a<n}$ is log-convex, as required.

Next, we consider the relationships between clan probabilities under the two models. For instance, consider the ratio of $p_{n}^{*}(a) / q_{n}^{*}(a)$ with $n=30$ (see Figure 4 . Then the ratios are symmetric about $a=15$, which is consistent with Part(i) in Theorem 12. In addition, by the figure it is clear that, except for $a=1$ for which $p_{n}^{*}(a)=q_{n}^{*}(a)=1$, the ratio is strictly decreasing on $[2,\lfloor n / 2\rfloor]$ and is less than 1 when $a$ is greater than a critical value. We shall show this observation holds for general $n$. To this end, we need the following technical lemma.

Lemma 5 For $n>5$, we have $p_{n}^{*}(\lfloor n / 2\rfloor)<q_{n}^{*}(\lfloor n / 2\rfloor)$.

Proof For simplicity, let $k=\lfloor n / 2\rfloor$. To establish the lemma, we consider the following two cases. 
The first case is when $n$ is even, that is, $n=2 k$. Then we have

$$
\begin{aligned}
p_{2 k}^{*}(k) & =4 k\left(\frac{2}{k(k+1)}-\frac{1}{2 k(2 k-1)}\right)\left(\begin{array}{c}
2 k \\
k
\end{array}\right)^{-1} \\
& =\left(\frac{8}{k+1}-\frac{2}{2 k-1}\right)\left(\begin{array}{c}
2 k \\
k
\end{array}\right)^{-1}=\frac{2(7 k-5)}{(k+1)(2 k-1)}\left(\begin{array}{c}
2 k \\
k
\end{array}\right)^{-1},
\end{aligned}
$$

and

$$
\begin{aligned}
\alpha(k):=\frac{q_{2 k}^{*}(k)}{p_{2 k}^{*}(k)} & =\frac{\varphi(k) \varphi(k)}{\varphi(2 k-1)}\left(\begin{array}{c}
2 k \\
k
\end{array}\right) \frac{(k+1)(2 k-1)}{2(7 k-5)} \\
& =\frac{(2 k-2) !(2 k-2) !(2 k-2) !(2 k) !}{(4 k-4) !(k-1) !(k-1) ! k ! k !} \frac{(k+1)(2 k-1)}{2(7 k-5)}
\end{aligned}
$$

Note that $\alpha(3)=\frac{15}{14}>1$, and $\alpha(k)$ is increasing for $k \geqslant 3$, because

$$
\begin{aligned}
\frac{\alpha(k+1)}{\alpha(k)} & =\frac{2(2 k-1)(2 k+1)^{2}(k+2)(7 k-5)}{(4 k-1)(4 k-3)(k+1)^{2}(7 k+2)} \\
& =\frac{112 k^{5}+200 k^{4}-116 k^{3}-130 k^{2}+22 k+20}{112 k^{5}+144 k^{4}-59 k^{3}-96 k^{2}+k+6} \\
& >1
\end{aligned}
$$

holds for $k \geqslant 3$. In other words, for $k \geqslant 3$, we have $\alpha(k)>1$ and hence also $q_{2 k}^{*}(k)>p_{2 k}^{*}(k)$.

The second case is when $n$ is odd, that is, $n=2 k+1$. Then we have

$$
\begin{aligned}
p_{2 k+1}^{*}(k) & =(4 k+2)\left(\frac{1}{k(k+1)}+\frac{1}{(k+1)(k+2)}-\frac{1}{2 k(2 k+1)}\right)\left(\begin{array}{c}
2 k+1 \\
k
\end{array}\right)^{-1} \\
& =\frac{7 k+2}{k(k+2)}\left(\begin{array}{c}
2 k+1 \\
k
\end{array}\right)^{-1},
\end{aligned}
$$

and

$$
\begin{aligned}
\beta(k):=\frac{q_{2 k+1}^{*}(k)}{p_{2 k+1}^{*}(k)} & =\frac{\varphi(k) \varphi(k+1)}{\varphi(2 k)}\left(\begin{array}{c}
2 k+1 \\
k
\end{array}\right) \frac{k(k+2)}{7 k+2} \\
& =\frac{(2 k-2) !(2 k-1) !(2 k) !(2 k+1) !(k+2)}{(4 k-2) !(k-1) !(k-1) ! k !(k+1) !(7 k+2)} .
\end{aligned}
$$

Now we have $\beta(3)=25 / 23>1$. In addition, $\beta(k)$ is increasing for $k \geqslant 3$ by noting that

$$
\begin{aligned}
\frac{\beta(k+1)}{\beta(k)} & =\frac{(2 k-1)(2 k+1)(2 k+2)(2 k+3)(k+3)(7 k+2)}{(k+2)^{2}(4 k+1)(4 k-1) k(7 k+9)} \\
& =\frac{112 k^{6}+648 k^{5}+1156 k^{4}+630 k^{3}-152 k^{2}-198 k-36}{112 k^{6}+592 k^{5}+1017 k^{4}+539 k^{3}-64 k^{2}-36 k} \\
& \geqslant 1
\end{aligned}
$$

holds for $k \geqslant 3$. In other words, for $k \geqslant 3$ and $n$ being odd, we also have $\beta(k)>1$ and hence also $q_{2 k+1}^{*}(k)>p_{2 k+1}^{*}(k)$. This completes the proof.

Parallel to Theorem 5 which compares $p_{n}(a)$ and $q_{n}(a)$, the following theorem provides a comparison between $p_{n}^{*}(a)$ and $q_{n}^{*}(a)$.

Theorem 13 For $n>5$, there exists a number $\kappa^{*}(n)$ in $(1,\lfloor n / 2\rfloor)$, such that $p_{n}^{*}(a)>q_{n}^{*}(a)$ for $2 \leqslant a \leqslant \kappa^{*}(n)$, and $p_{n}^{*}(a)<q_{n}^{*}(a)$ for $\kappa^{*}(n)<a \leqslant\lfloor n / 2\rfloor$. 
Proof For simplicity, let $b:=n-a$. Since we have

$$
p_{n}^{*}(2)=\frac{4\left(\frac{1}{6}+\frac{2}{n(n-1)(n-2)}\right)}{n-1}>\frac{2}{3(n-1)} \geqslant \frac{1}{2 n-5}=q_{n}^{*}(2),
$$

and $p_{n}^{*}(\lfloor n / 2\rfloor)<q_{n}^{*}(\lfloor n / 2\rfloor)$ by Lemma 5 , it suffices to prove that

$$
g_{n}(a)=\frac{p_{n}^{*}(a)}{q_{n}^{*}(a)}
$$

is strictly decreasing on $[2,\lfloor n / 2\rfloor]$. To this end, let

$$
f_{n}(a)=\frac{1}{a(a+1)}+\frac{1}{b(b+1)}-\frac{1}{n(n-1)} .
$$

From the definition of $g_{n}(a)$ and Theorem 11, we have

$$
\frac{g_{n}(a+1)}{g_{n}(a)}=\frac{f_{n}(a+1)}{f_{n}(a)} \frac{(a+1)(2 b-3)}{b(2 a-1)},
$$

which is less than 1 for $2 \leqslant a \leqslant\lfloor n / 2\rfloor-1$ if and only if

$$
\beta_{n}(a):=f_{n}(a) b(2 a-1)-f_{n}(a+1)(a+1)(2 b-3)>0 \quad \text { for } 2 \leqslant a \leqslant\lfloor n / 2\rfloor-1 .
$$

In the rest of the proof, we shall establish Eq. (17). To begin with, note that

$$
\begin{array}{r}
\beta_{n}(a)=\frac{3}{n-1}-\frac{3(2 a+1)}{n(n-1)}+\frac{2 a^{2}+a n+5 a-2 n}{a(a+1)(a+2)} \\
+\frac{2 a-3 n}{(b-1)(b+1)}+\frac{a+2 n+3}{(b-1) b(b+1)} .
\end{array}
$$

This implies

$$
\begin{aligned}
\beta_{n}(2) & =\frac{3 n^{4}-18 n^{3}-39 n^{2}+342 n-360}{4 n(n-1)(n-2)(n-3)} \\
& =\frac{3 n^{2}\left(n^{2}-6 n-13\right)+(342 n-360)}{4 n(n-1)(n-2)(n-3)}>0
\end{aligned}
$$

for $n \geqslant 6$ because $\beta_{6}(2)=1 / 5, \beta_{7}(2)=24 / 70$ and $n^{2}-6 n-13>0$ for $n \geqslant 8$. In addition, we have

$$
\beta_{2 t+1}(t)=\frac{4 t^{2}+2 t-2}{t(t+1)(t+2)}+\frac{-4 t+2}{t(t+2)}+\frac{5}{t(t+2)}>0
$$

for $t \geqslant 3$ and

$$
\begin{aligned}
\beta_{2 t+2}(t) & =\frac{3}{2 t-1}-\frac{3}{2 t+2}+\frac{4 t^{2}+3 t-4}{t(t+1)(t+2)}-\frac{4 t+6}{(t+1)(t+3)}+\frac{(5 t+7)}{(t+1)(t+2)(t+3)} \\
& =\frac{9}{(2 t-1)(2 t+2)}+\frac{6 t^{2}-12}{t(t+1)(t+2)(t+3)} \\
& >0
\end{aligned}
$$

for $t \geqslant 2$. Therefore, we have $\beta_{n}(\lfloor n / 2\rfloor-1) \geqslant 0$ for $n \geqslant 6$.

It remains to show that $\beta_{n}(a)$ is strictly decreasing, that is, $\beta_{n}(a)-\beta_{n}(a+1)>0$ for $3 \leqslant a \leqslant\lfloor n / 2\rfloor-1$. Indeed, by Eqn. (18) we have

$$
\begin{aligned}
\beta_{n}(a)-\beta_{n}(a+1) & =\frac{6}{n(n-1)}+\frac{2 a^{2}+2 a n+8 a-6 n}{a(a+1)(a+2)(a+3)}+\frac{2 a^{2}-6 a n+4 n^{2}-10 n-8}{(b-2)(b-1) b(b+1)} \\
& >\frac{n^{2}-7 n-8+2 a^{2}}{(b-2)(b-1) b(b+1)} \\
& >0 .
\end{aligned}
$$


Here the first inequality follows from $a \geqslant 3$ and $a \leqslant\lfloor n / 2\rfloor-1 \leqslant(n-1) / 2$ implying $3 n^{2}-6$ an $\geqslant 3 n$, and the second one from $a \geqslant 3$ and $n \geqslant 6$. This completes the proof.

We end this section with some correlation results about clan probabilities under the PDA model.

Theorem 14 Let $A_{1}, \ldots, A_{k}$ be $k$ disjoint (nonempty) subsets of $X$, and let $m=\left|A_{1}\right|+\cdots+\left|A_{k}\right|$. Then we have

$$
\mathbb{P}_{u P D A}\left(A_{1}, \ldots, A_{k}\right)=\frac{\varphi(n-m+k-1) \prod_{i=1}^{k} \varphi\left(\left|A_{i}\right|\right)}{\varphi(n-1)} .
$$

Proof Since $\mathbb{P}_{\mathrm{uPDA}}\left(T^{*}\right)=1 / \varphi(n-1)$ for each tree $T^{*}$ in $\mathscr{T}_{X}$, it remains to compute the number of trees that have $A_{1}, \ldots, A_{k}$ as clans is $\varphi(n-m+k-1) \prod_{i=1}^{k} \varphi\left(\left|A_{i}\right|\right)$. To this end, note that such a tree can be constructed in two steps:

1. Build an unrooted tree on $\left(X \backslash \bigcup_{i=1}^{k} A_{i}\right) \cup\left\{x_{1}, \ldots, x_{k}\right\}$, where $x_{1}, \ldots, x_{k}$ are leaves not in $X$ serving as "placeholders" used in the second step.

2. Replace each $x_{i}$ with a tree in $\mathscr{T}_{i}$.

There are $\varphi(n-m+k-1)$ different choices for a tree in the first step, and there are $\prod_{i=1}^{k} \varphi\left(a_{i}\right)$ different ways to replace $x_{1}, \ldots, x_{k}$ by trees in $\mathscr{T}_{A_{1}}, \ldots, \mathscr{T}_{A_{k}}$. The claim then follows.

Theorem 15 Let $A$ and $B$ be two subsets of $X$ with $a \leqslant b$, where $a=|A|$ and $b=|B|$. Then we have

$$
\mathbb{P}_{u P D A}(A, B)= \begin{cases}\frac{\varphi(b) \varphi(n-b) \varphi(a) \varphi(b-a)}{\varphi(n-1) \varphi(b-1)}, & \text { if } A \subseteq B, \\ \frac{\varphi(a) \varphi(b) \varphi(n-a-b+1)}{\varphi(n-1)}, & \text { if } A \text { and } B \text { are disjoint } \\ 0, & \text { otherwise. }\end{cases}
$$

Proof The first case follows by applying Theorem 11 twice; the second case follows from Theorem 14.

Corollary 3 Let $A$ and $B$ be two compatible subsets of $X$. Then we have

$$
\mathbb{P}_{u P D A}(A, B) \geqslant \mathbb{P}_{u P D A}(A) \mathbb{P}_{u P D A}(B)
$$

Proof Set $a=|A|$ and $b=|B|$. By symmetry we may assume without loss of generality that $a \leqslant b$ holds. Since $A$ and $B$ are compatible, we have either $A \cap B=\emptyset$ or $A \subseteq B$.

To establish the theorem for the first case, note first that $n-a-b+1 \leqslant n-b \leqslant n-a \leqslant n-1$ holds. Therefore by Lemma 3, we have

$$
\varphi(n-a-b+1) \varphi(n-1) \geqslant \varphi(n-a) \varphi(n-a),
$$

and hence

$$
\frac{\varphi(a) \varphi(b) \varphi(n-a-b+1)}{\varphi(n-1)} \geqslant\left(\frac{\varphi(b) \varphi(n-b)}{\varphi(n-1)}\right)\left(\frac{\varphi(a) \varphi(n-a)}{\varphi(n-1)}\right) .
$$

Together with Theorem 15, this shows that the theorem holds for the case $A \cap B=\emptyset$.

For the second case, note that $b-a \leqslant n-a \leqslant n-1$ and $b-a \leqslant b-1 \leqslant n-1$ hold. Therefore by by Lemma 3, we have

$$
\varphi(n-1) \varphi(b-a) \geqslant \varphi(b-1) \varphi(n-a)
$$

and hence

$$
\frac{\varphi(b) \varphi(n-b) \varphi(a) \varphi(b-a) \varphi(n-b)}{\varphi(n-1) \varphi(b-1)} \geqslant\left(\frac{\varphi(b) \varphi(n-b)}{\varphi(n-1)}\right)\left(\frac{\varphi(a) \varphi(n-a)}{\varphi(n-1)}\right) .
$$

Together withTheorem 15, this shows that the theorem holds for the case $A \subseteq B$, as required. 


\section{Discussion and concluding remarks}

Clade sizes are an important genealogical feature in the study of phylogenetic and population genetics. In this paper we present a comparison study between the clade probabilities under the YHK and PDA models, two null models which are commonly used in evolutionary biology.

Our first main result reveals a common feature, that is, the clade probability sequences are log-convex under both models. This implies that compared with 'mid-sized' clades, very 'large' clades and very 'small' clades are more likely to occur under these two models, and hence provides a theoretical explanation for the empirical result on the PDA model observed by Pickett and Randle (2005). One implication of this result is that in Bayesian analysis where the two null models are used as prior distribution, the distribution on clades is not uninformative as bias is given to those whose sizes are extreme. Therefore, further considerations or adjustment, such as introducing a Bayes factor to account for the bias on prior clade probabilities, is important to interpret posterior Bayesian clade supports.

The second result reveals a 'phase transition' type feature when comparing the sequences of clade probabilities under the two null models. That is, we prove that there exists a critical value $\kappa(n)$ such that the probability that a given clade with size $k$ is contained in a random tree with $n$ leaves generated under the YHK model is smaller than that under the PDA model for $1<k \leqslant \kappa(n)$, and higher for all $\kappa(n) \leqslant k<n$. This implies that typically the trees generated under the YHK model contains relatively more 'small' clades than those under the PDA model.

The above two results are also extended to unrooted trees by considering the probabilities of 'clans', the sets of taxa that are all on one side of an edge in an unrooted phylogenetic tree. This extension is relevant because in many tree reconstruction approaches, the problem of finding the root is either ignored or left as the last step. Here we study the sequences formed by clan probabilities for unrooted trees generated by the two null models, and obtain several results similar to those for rooted trees.

Note that the two models studied here are special instances of the $\beta$-splitting model introduced by Aldous (1996), a critical branching process in which the YHK model corresponds to $\beta=0$ and the PDA model to $\beta=-1.5$. Therefore, it would be of interest to study clade and clan probabilities under this more general model. In particular, it is interesting to see whether the relationships between two models revealed in this paper also hold for general $\beta$.

Acknowledgements We thank Prof. Kwok Pui Choi and Prof. Noah A. Rosenberg for simulating discussions and useful suggestions. We would also like to thank two anonymous referees for their helpful and constructive comments on the first version of this paper.

\section{References}

Agapow PM, Purvis A (2002) Power of eight tree shape statistics to detect nonrandom diversification: a comparison by simulation of two models of cladogenesis. Systematic Biology 51:866-872

Aldous D (1996) Probability distributions on cladograms. In: Aldous D, Pemantle R (eds) Random Discrete Structures, The IMA Volumes in Mathematics and its Applications, vol 75, Springer-Verlag, pp 1-18

Aldous D (2001) Stochastic models and descriptive statistics for phylogenetic trees, from Yule to today. Statistical Science 16(1):23-34

Blum MGB, Francois O (2005) Minimal clade size and external branch length under the neutral coalescent. Advances in Applied Probability 37:647662

Blum MGB, Francois O, Janson S (2006) The mean, variance and limiting distribution of two statistics sensitive to phylogenetic tree balance. The Annals of Applied Probability 16(4):2195-2214

Brown JKM (1994) Probabilities of evolutionary trees. Systematic Biology 43(1):78-91 
Colless DH (1982) Review of "Phyogenetics: The theory and practice of phylogenetic systematics". Systematic Zoology 31:100-104

Felsenstein J (2004) Inferring Phylogenies. Sinauer Associates, Sunderland, MA

Harding EF (1971) The probabilities of rooted tree-shapes generated by random bifurcation. Advances in Applied Probability 3(1):44-77

Heard SB (1992) Patterns in tree balance among cladistic, phenetic, and randomly generated phylogenetic trees. Evolution 46(6):1818-1826

Hudson RR, Coyne JA (2002) Mathematical consequences of the genealogical species concept. Evolution 56(8):1557-1565

Kingman JFC (1982) On the genealogy of large populations. Journal of Applied Probability 19:27-43

Li S, Pearl DK, Doss H (2000) Phylogenetic tree construction using Markov Chain Monte Carlo. Journal of the American Statistical Association 95(450):493-508

Liu LL, Wang Y (2007) On the log-convexity of combinatorial sequences. Advances in Applied Mathematics 39:453-476

McKenzie A, Steel MA (2000) Distributions of cherries for two models of trees. Mathematical Biosciences 164:81-92

Mooers AO, Heard SB (1997) Evolutionary process from phylogenetic tree shape. Quarterly Review of Biology 72:31-54

Mooers AO, Heard SB (2002) Using tree shape. Systematic Biology 51:833-834

Nordborg M (1998) On the probability of Neanderthal ancestry. American Journal of Human Genetics 63:1237-1240

Nordborg M (2001) Coalescent theory. In: Balding DJ, Bishop M, Cannings C (eds) Handbook of Statistical Genetics, Wiley, Chichester, UK, chap 7, pp 179-212

Pickett KM, Randle CP (2005) Strange bayes indeed: uniform topological prior imply non-uniform clade priors. Molecular Phylogenetics and Evolution 34:203-211

Pinelis I (2003) Evolutionary models of phylogenetic trees. Proceedings of the Royal Society of London Series B: Biological Sciences 270:1425-1431

Rannala B, Yang Z (1996) Probability distribution of molecular evolutionary trees: a new method of phylogenetic inference. Journal of Molecular Evolution 43:304-311

Rogers JS (1996) Central moments and probability distributions of three measures of phylogenetic tree imbalance. Systematic Biology 45:99-110

Rosenberg NA (2003) The shapes of neutral gene genealogies in two species: probabilities of monophyly, paraphyly and polyphyly in a coalescent model. Evolution 57(7):1465-1477

Rosenberg NA (2006) The mean and variance of the numbers of r-pronged nodes and r-caterpillars in Yule-generated genealogical trees. Annals of Combinatorics 10:129-146

Rosenberg NA (2007) Statistical tests for taxonomic distinctiveness from observations of monophyly. Evolution 61(2):317-323

Sackin MJ (1972) "Good" and "bad" phenograms. Systematic Zoology 21(2):225-226

Semple C, Steel MA (2003) Phylogenetics. Oxford University Press, Oxford, UK

Steel M, Pickett KM (2006) On the impossibility of uniform priors on clades. Molecular Phylogenetics and Evolution 39:585-586

Steel MA (2012) Root location in random trees: A polarity property of all sampling consistent phylogenetic models except one. Molecular Phylogenetics and Evolution 65(1):345 - 348

Yule GU (1925) A mathematical theory of evolution. based on the conclusions of Dr. J.C. Willis, F.R.S. In: Philosophical Transactions of the Royal Society of London. Series B, Containing Papers of a Biological Character, vol 213, The Royal Society, pp 21-87

Zhu S, Degnan JH, Steel MA (2011) Clades, clans and reciprocal monophyly under neutral evolutionary models. Theoretical Population Biology 79:220-227 